\title{
Special Section Guest Editorial: High-Performance Computing in Applied Remote Sensing
}

\author{
Bormin Huang a and Antonio Plaza \\ ${ }^{a}$ University of Wisconsin-Madison, Space Science and Engineering Center, \\ 1225 West Dayton Street, Madison, Wisconsin 53706 \\ bormin@ssec.wisc.edu \\ ${ }^{\mathrm{b}}$ University of Extremadura, Hyperspectral Computing Laboratory, \\ Avda. de la Universidad s/n E-10003 Caceres, Spain
}

This special section on high-performance computing in applied remote sensing presents the state-of-the-art research in incorporating high-performance computing (HPC) facilities and algorithms for effective and efficient remote sensing applications. Technological advances in modern active and passive sensors with higher spectral, spatial, and/or temporal resolutions have resulted in a substantial increase in multidimensional data volume. The increase poses a challenge to processing remote sensing data in a more timely fashion for environmental, commercial, or military applications. Parallel, distributed, and grid computing facilities and algorithms have become indispensable tools to tackle the issues of processing massive remote sensing data. In recent years, the graphics processing unit (GPU) has evolved into highly parallel many-core processor with tremendous computing power and high memory bandwidth to offer two to three orders of magnitude speedup over the central processing unit (CPU). A cost-effective GPU-based computer has become an appealing alternative to an expensive CPU-based computer cluster for many researchers performing various scientific and engineering applications.

$1931-3195 / 2011 / \$ 25.00$ @ 2011 SPIE 